\section{$\underset{\substack{\text { hommes } \\ \text { \& migrations }}}{ }$}

\section{Hommes \& migrations}

Revue française de référence sur les dynamiques

migratoires

1286-1287 | 2010

Les migrations subsahariennes

\title{
Territorialités en mouvement
}

Migration, décentralisation, développement dans la région de Kayes

(Mali)

\section{Stéphanie Lima}

\section{(2) OpenEdition \\ Journals}

\section{Édition électronique}

URL : http://journals.openedition.org/hommesmigrations/1762

DOI : 10.4000/hommesmigrations. 1762

ISSN : 2262-3353

\section{Éditeur}

Musée national de l'histoire de l'immigration

\section{Édition imprimée}

Date de publication : 1 juillet 2010

Pagination : 258-267

ISSN : 1142-852X

Référence électronique

Stéphanie Lima, «Territorialités en mouvement », Hommes \& migrations [En ligne], 1286-1287 | 2010, mis en ligne le 29 mai 2013, consulté le 03 mai 2019. URL : http://journals.openedition.org/ hommesmigrations/1762 ; DOI : 10.4000/hommesmigrations.1762 


\section{Territorialités en mouvement Migration, décentralisation, développement dans la région de Kayes (Mali)}

Par Stéphanie Lima, maître de conférences en géographie, CUFR JF Champollion - Albi, UMR Dynamiques rurales - université de Toulouse

Au Mali, le lien social se joue des distances et excède le territoire national. Regroupés en associations à l'étranger, les migrants maliens restent fortement connectés à leur village d'origine. L'État malien s'est appuyé sur leurs réseaux dans le cadre de la politique de décentralisation menée dans les années quatre-vingt-dix. Le nouveau découpage communal risquant de susciter des rivalités entre les territoires, les migrants apparaissent comme des médiateurs incontournables entre les autorités publiques et les populations locales. 
Depuis 1991, le Mali, grand pays d'Afrique de l'Ouest, s'est ouvert au monde, après plusieurs décennies de dictature militaire, et a pris le chemin de la démocratie. Dès 1992, il entreprend un vaste chantier, celui de la décentralisation, sous les recommandations des instances internationales et sous les exhortations de la population dont la parole est désormais libérée. La mise en ceuvre de la décentralisation s'accompagne d'une réorganisation territoriale au niveau local, avec la création de communes, rurales et urbaines, en remplacement des arrondissements, découpage hérité de l'indépendance du pays au début des années soixante. L'entreprise de la communalisation est peu banale, car plutôt que de reposer sur une logique descendante et unilatérale, elle associe la "base", puisque la population est partie prenante du projet. Ainsi, loin de pouvoir, ni de vouloir imposer un maillage, l'État a proposé des critères sur lesquels l'ensemble des Maliennes et Maliens étaient appelés à se prononcer en vue de l'émergence des territoires communaux. Les communes rurales, issues du regroupement de plusieurs villages dont un est le chef-lieu, se présentent comme l'aboutissement d'une rencontre inattendue et improbable. En effet, d'un côté, l'État compose une palette de critères dont la synthèse relève de l'utopie de "l'optimum territorial" et, de l'autre, les populations projettent le dessin de figures spatiales ancrées dans le temps long, celui des anciens "pays" précoloniaux et des réseaux sociaux aux ancrages discontinus dans l'espace. Au terme de deux années de concertations intervillageoises, la configuration des territoires communaux exprime cette tension entre territorialité fonctionnelle et territorialité sociale, à laquelle s'ajoute la dimension réticulaire de l'espace social, complètement intégrée dans la construction communale. La fabrique des territoires communaux s'est heurtée à des espaces mobiles, en témoigne l'importance des réseaux sociaux qui se déploient à toutes les échelles, depuis les liens entre localités villageoises qui se reconnaissent une origine commune, jusqu'aux réseaux migratoires qui unissent les migrants à leur village d'origine, quel que soit leur lieu de résidence. Ainsi, les communes, enveloppes institutionnelles négociées au plus près des espaces sociaux, sont des formations hybrides produites entre distance sociale et distance spatiale. Le couple espace-distance se révèle comme le moteur de la territorialisation en alliant les impératifs des interactions de proximité spatiale aux nécessités des relations réticulaires entre des lieux discontinus, insérés quant à eux dans un système de dépendance sociale. Ainsi la place des réseaux dans la création des territoires communaux n'apparaît pas comme un fait exceptionnel mais plutôt comme une condition essentielle de leur réalisation.

Les associations de migrants maliens, originaires de la région de Kayes et résidant en France, sont au cceur de ce mouvement. Depuis plusieurs décennies, elles entretiennent des relations exclusives avec leurs villages d'origine, ces liens conduisant à des innovations spatiales en termes de recompositions territoriales. Aujourd'hui, les 
communes concernées par la dynamique migratoire dans le nord de la région de Kayes portent l'empreinte de ces réseaux, tant dans leur genèse que dans leur fonctionnement présent. En effet, les associations n'ont eu de cesse d'être présentes dans le processus de communalisation, d'une part, et de participer à la vie communale, d'autre part. Elles portent en elles cette utopie, celle des réseaux en partage dans l'espace, entre lien et distance, et ce au-delà du découpage communal, porteur de nouvelles discontinuités à l'échelle locale.

\section{Réseaux et constructions territoriales : le point de vue des migrants}

Dès 1994, la Mission de décentralisation(MDD) ${ }^{11}$, par l'intermédiaire du responsable de la Cellule de "Réorganisation territoriale", s'est intéressée à l'implication des associations de ressortissants de la région de Kayes en France dans l'application de la décentralisation et dans la mise en ceuvre du découpage communal. Entre 1994 et 1998, quatre missions se sont succédé. Elles ont permis à la MDD d'informer régulièrement les membres de la communauté malienne en France sur l'état d'avancement de la communalisation et d'échanger sur les rôles et les prérogatives des associations dans le nouveau contexte suscité par cette politique.

La première rencontre entre les associations et la Mission de décentralisation a eu lieu en novembre 1994. Il s'agissait de commencer à sensibiliser les associations, considérées comme des interlocutrices incontournables pour la mise en ceuvre de la décentralisation, et notamment de son volet territorial. Ces premiers échanges ont suscité de nombreuses questions de la part des associations: sur l'avenir de la migration; sur le poids de l'administration, jugée trop présente concernant les initiatives des associations ; sur la gestion de l'espace par les populations ; etc. À ce stade des débats, les interrogations portaient sur les changements provoqués par la décentralisation vis-à-vis de l'ancien système administratif, et sur les avantages de la réforme. Les questions sur l'articulation des compétences et des pouvoirs entre associations et communes interviendront plus tard quand, une fois l'enjeu territorial négocié, se présentera l'enjeu électoral avec la composition des listes pour le scrutin municipal.

Lors de cette réunion, l'accent a été mis sur les modalités de création des territoires communaux. La position des migrants à ce point des opérations concernant l'orientation du découpage était de suivre l'avis des villageois sur place, dans la mesure où leurs choix ne portaient pas préjudice à la structure associative. Les représentations spatiales des migrants sollicitées pour imaginer les contours des futurs territoires 
communaux allaient dans le même sens que celles des villageois, avec une importance accordée aux réalités foncières, à la primauté historique des villages mères et aux statuts sociaux. Cependant, le découpage territorial a été perçu par d'autres comme le moyen pour les petits villages de s'affranchir de la tutelle de villages anciens ou de leur village mère, et ce d'autant plus s'ils répondaient de leur côté à des critères fonctionnels (infrastructures). Cette dichotomie naissante dans la lecture des critères du découpage s'est développée par la suite, avec pour conséquences des ruptures entre terroirs villageois voisins. Si les enjeux du découpage n'ont pas été entièrement saisis lors de cette première concertation, c'est au fur et à mesure des rencontres en France, mais aussi des contacts réguliers entre les migrants et leur village, que le positionnement des associations va se faire, avec des interactions différentes.

\section{La réactivation des clivages entre territoires}

En janvier 1996, la MDD effectue une deuxième mission auprès des associations pour les informer de l'état d'avancement du découpage communal dans la région de Kayes. Il s'agit aussi de recueillir leurs réactions sur ces propositions et d'échanger sur des points d'ordre général à propos de la décentralisation. C'est à ce moment-là que les migrants commencent à réagir plus ouvertement et à s'exprimer sur le dessin des futures communes. 
En deux années, la décentralisation s'est concrétisée, et les premières images du découpage font en quelque sorte l'effet d'un "électrochoc" dans la communauté des migrants. Jusqu'alors, ceux-ci avaient adopté une position attentiste, ne sachant pas si l'État parviendrait à aller jusqu'au bout de sa démarche. Le réveil provoqué par les premières tendances de la réorganisation terri-

Face aux changements annoncés, les premières réactions des migrants en France sont teintées d'inquiétude et de méfiance par rapport à la politique de décentralisation et au projet de réorganisation territoriale. toriale n'a pas été des plus sereins pour tous. D'après le responsable de la Cellule de "Réorganisation territoriale" (entretien, Bamako, 2000), migrants et villageois étaient aussi partagés sur la nature des territoires communaux. Ainsi, malgré la proximité de certaines localités, les différences de statut sont source de mésentente et parfois de rupture, notamment au moment du choix du chef-lieu de la commune. Selon lui, "les

migrants ne peuvent pas brader la richesse foncière de leur village et ils n'acceptent pas que des localités qui ont moins de cinquante ans d'existence puissent avoir des responsabilités communales, ou deviennent le centre de la commune". Et comme le confirme un responsable associatif (entretien, Saint-Denis, 2001), "le découpage a été un moment fort de débat et de conflit, au cours duquel les vieux clivages sont ressortis". Mais, encore une fois, cette lecture "traditionnelle" de l'espace n'est pas partagée par toutes les associations, qu'elles soient villageoises ou intervillageoises.

D'après les entretiens effectués avec les acteurs du découpage dans la région de Kayes et avec les responsables associatifs en France, il ressort que les réactions des migrants ont été plus fortes dans le cas des associations qui n'ont pas de double structure, entre ici et là-bas, et plus particulièrement celles d'envergure villageoise. Ceci s'explique par la nature du lien qui unit un village et son village-bis en France : il semblerait qu'il soit beaucoup plus fort et unilatéral que dans le cas des associations intervillageoises et de celles qui ont des structures autonomes dans les villages, pour lesquelles il existe des pratiques de concertation, et de prise de décision partagées entre les deux espaces. Ainsi, les responsables d'associations en France qui ont des structures locales affirment qu'ils sont restés en retrait pendant les opérations de découpage, cette position ayant été ou non suivie par les structures locales dans les villages mêmes. C'est le degré d'autonomie entre les villages et les associations de migrants qui a joué, expliquant l'existence ou l'absence d'implication de ces derniers dans les opérations de découpage.

Après le vote de la loi "portant création de communes" en novembre 1996, une troisième mission de la MDD a été organisée, en décembre 1996, pour restituer les 
résultats du découpage au niveau des associations et entamer une série d'échanges sur les perspectives de l'implication des associations dans les collectivités territoriales. Car, une fois la question des territoires tranchée, c'est la mise en place des conseils communaux qui a attiré l'attention de la MDD sur le rôle que pouvaient tenir les migrants dans le processus électoral, notamment au niveau du choix des candidats et de la composition des listes. Ainsi, il s'agissait de "sensibiliser les migrants, pour qu'ils sensibilisent à leur tour leurs parents à propos des élections et du choix de candidats crédibles pour composer les listes des conseillers communaux" (entretien, Bamako, 2000). Les migrants apparaissent donc comme des interlocuteurs incontournables et, malgré leur éloignement, leur capacité d'intervention dans les villages est plus que jamais reconnue puisqu'ils sont interpellés en tant qu'intermédiaires entre "leurs parents" au village et l'État.

\section{Renversement des réseaux et proximité}

Si les migrants ont "fait la décentralisation avant l'État", comme certains le soulignent, la réforme et le découpage communal apportent des bouleversements de plusieurs ordres pour leurs associations. D'une part, les villages d'origine sont intégrés dans un territoire communal dans lequel les questions de développement se posent à l'échelle intervillageoise et, d'autre part, l'émergence de nouveaux pouvoirs communaux soumet les associations à un cadre de concertation tout autre, comparé à celui de la chefferie villageoise.

Face aux changements annoncés, les premières réactions des migrants en France sont teintées d'inquiétude et de méfiance par rapport à la politique de décentralisation et au projet de réorganisation territoriale. Lors d'un entretien, un responsable associatif déclarait ainsi : "Au départ, on aurait pu faire des tests plutôt que de gérer 701 communes. Dans un premier temps, il aurait peut-être mieux valu conserver les arrondissements en communes, et procéder par étapes successives. La décentralisation est une bonne chose, que les migrants ont déjà commencée. [...] Les associations ne vont pas financer la politique communale de l'État, même s'il y a un partenariat entre les associations et les communes. Mais il est hors de question de financer la commune en tant que telle. [...] Les associations se sont déjà substituées à l'État, il ne faut pas continuer dans le cas des communes. Les communes sont une bonne chose, mais elles sont comme des coquilles vides, sans moyens" (entretien, Paris, 2001). Ce témoignage exprime un positionnement assez tranché sur la réforme de décentralisation. Certes, les associations ont pris en charge plusieurs secteurs d'activités dans leurs villages 
d'origine, en dehors des réseaux administratifs locaux. Cependant, la création des collectivités territoriales correspond à une politique de l'État, vis-à-vis duquel les migrants ont une attitude tiraillée entre un besoin de reconnaissance et une volonté d'indépendance. Car, si les habitants de la région de Kayes se sentent "abandonnés" par l'État, ils ont su aussi attirer son attention, la dynamique migratoire étant une manière de pallier une situation de crise (sécheresse, enclavement, etc.) et en même temps d'attirer l'attention des pouvoirs publics ${ }^{(2)}$.

Au moment où l'État met en ceuvre la décentralisation, les associations optent, dans un premier temps, pour une stratégie attentiste. De France, un clivage s'est peu à peu constitué entre les structures à la démarche interventionniste et celles qui se sont positionnées en retrait de la réorganisation territoriale. Ainsi, plusieurs logiques se sont affirmées lors des concertations villageoises, en fonction des rapports de force entre les différents groupes sociaux à l'échelle villageoise et intervillageoise. Les commissions locales de découpage et les habitants eux-mêmes ont trouvé dans ces regroupements associatifs des "embryons" communaux correspondant aux critères fonctionnels. Les réseaux tissés entre ces deux composantes spatiales discontinues que sont les villages et les villages-bis, parfois synonymes de cloisonnement local, si ce n'est de "localisme", ont entraîné des mouvements de recomposition territoriale en-deçà des critères, en vue de maintenir un "entre-soi" configuré sur le mode réticulaire.

\section{Le pouvoir de médiation des associations de migrants}

Sur le terrain, l'existence des associations avait permis à certains villages de se soustraire de l'influence de voisins jugés trop imposants. Ainsi, les rapports de force entre les villages ont été révélés dans le processus de découpage communal, ce qui a contribué à la multiplication du nombre de communes créées. En dépit de liens historiques et sociaux reconnus entre deux villages, les mouvements de rupture ont été courants. L'enjeu du pouvoir est au cceur de ces événements, et les rapports entre les groupes sociaux, qui se déploient sur deux espaces différents, ajoutent à la complexité des interrelations qui interviennent dans le découpage communal. Les épiphénomènes territoriaux révèlent un aspect de ces tensions qui se déroulent sur plusieurs niveaux, et articulent pressions sociales et représentations spatiales. Le découpage communal se présente comme une épreuve à surmonter pour chaque groupe qui entre en lice dans la compétition pour le pouvoir. Chacun a dû se positionner face à l'offre territoriale et plus particulièrement pour l'obtention du 
chef-lieu de commune. Des alliances anciennes ont été brisées, tandis que d'autres ont été renouvelées. De nouvelles solidarités ont émergé et des isolats se sont construits. La diversité des logiques du découpage s'inscrit dans des contextes locaux spécifiques. Les influences des associations de migrants apparaissent comme un facteur supplémentaire parmi les différents éléments composant l'écheveau des motivations développées à l'occasion des concertations intervillageoises et au moment des restitutions régionales et extra-territoriales (en France). Le barrage des critères n'a pas résisté à ces poussées, qu'elles se soient exercées localement (cercle de Kéniéba) ou depuis l'extérieur (cercle de Yélimané). Tout un chacun a donc cherché à utiliser ses réseaux, si nécessaire, pour défendre des intérêts territoriaux localisés. En cela, les villages demeurent au centre des relations réticulaires, quelles que soient leurs échelles.

Les réseaux constitués entre les villages et les associations de ressortissants ont marqué l'espace d'origine, l'ont transformé, ce qui n'est pas sans poser question dans le contexte de la réorganisation communale. Les structures associatives se présentent comme des compositions sociales et spatiales hybrides, à la croisée de plusieurs enjeux, dans la mesure où elles sont nées d'une pratique spatiale spécifique, la mobilité, qui s'est traduite par l'ouverture des terroirs villageois vis-à-vis de lieux éloignés. 


\section{Vers un partage de l'espace, entre lien et distance}

La mobilité est source de recompositions spatiales. Elle participe d'une territorialité renouvelée, empreinte d'anciens liens et de nouveaux réseaux. En cela, la construction des territoires locaux est le fait d'acteurs mobiles qui opèrent une mise en relation entre des espaces discontinus. Cependant, ce type de territorialité, fondé sur des espaces de vie fragmentés, pose le problème des modalités d'articulation à la fois au niveau local et entre espace local et espace national. Aujourd'hui, les associations de migrants sont dans une période de transition. Leur rôle dans le développement local s'est réduit depuis les années quatre-vingt-dix, qualifiées d'années "d'utopies (3)". Les relations privilégiées entre villages d'origine et communautés de ressortissants ont atteint les limites du fonctionnement en vase clos. Louverture est devenue nécessaire, bien qu'elle signifie un relâchement dans le système réticulaire. Les liens se sont parfois distendus entre les migrants et les villageois, au profit d'une association implantée localement, ce qui a permis le renforcement des liens intervillageois. La communalisation a accentué cette tendance, étant donné que la construction des territoires communaux repose sur le regroupement des terroirs villageois. Alors que pour ces villages de la région de Kayes, la constitution d'un réseau social entre plusieurs lieux discontinus avait débouché sur des investissements, ce que l'on peut appeler du "développement", la production d'un maillage institutionnel dépend de l'articulation de deux dynamiques spatiales que l'on présente souvent comme contradictoires. Les réseaux seraient contre les territoires et réciproquement. Pourtant, on peut poser l'hypothèse suivant laquelle les logiques réticulaire et territoriale peuvent s'accorder, au-delà du champ "local", et ce justement au nom du "développement". Le développement local étant un objectif promu à travers la décentralisation et pour lequel les communes forment le cadre de référence.

Dès lors, une question se pose : quelle est la dimension spatiale du développement? En effet, n'est-elle pas celle du champ d'intervention des acteurs sociaux qui s'engagent dans cette quête, suivant le principe selon lequel les limites sociales précèdent les limites spatiales? Dans ce sens, la mobilité se présente encore et toujours comme une ressource. Les expériences passées des associations de migrants, leur savoir-faire en matière d'articulation des espaces nous permettent de penser que les "gens du fleuve $e^{(4)}$ ' feront encore preuve d'imagination en termes d'innovations sociales et de pratiques spatiales. La communalisation représente-t-elle cette opportunité ?

Avec la communalisation, les associations de migrants et les associations locales ont vu nombre de leurs acquis être mis sur la sellette. Non seulement leur assise spatiale est remise en question, mais aussi leur capital humain (étant donné que plusieurs 
leaders associatifs sont devenus des élus communaux). Aujourd'hui, elles ont un défi à relever, celui de leur adaptation à la nouvelle donne communale, qui ouvre un champ de possibilités pour l'action associative malgré les contraintes adjacentes à sa création. En effet, un nouveau cadre de concertation s'offre aux associations, en rupture avec les tensions qui animaient jusqu'à présent les relations établies entre elles et l'État, notamment via les administrations régionales. Les associations sont désormais dans un espace de négociation différent, dans la mesure où leur engagement qui répondait aux carences de l'État n'est plus d'actualité. Leur position marquée, "contre" l'État, n'est plus pertinente dans la mesure où l'institution communale représente l'État d'un côté et les villages de l'autre. Le "contre" et "à la place de" l'État est appelé à se transformer en "travailler avec". De nouvelles voies se dessinent aujourd'hui en ce sens, et l'on peut se demander quels en sont les ressorts et les perspectives.

Si les associations avaient atteint certaines limites dans leurs actions auparavant par manque d'articulation à l'échelle locale et régionale, l'arrivée des communes pose aujourd'hui le problème de la concurrence entre ces deux entités. À plus long terme, la question est de savoir si la seule perspective des associations est de se mouler dans le cadre communal et d'être au service des élus municipaux, ou si elles auront les moyens de se reconstruire en tant qu'outil de développement. La place des associations, sous la forme qui a été la leur pendant les années quatre-vingt et quatrevingt-dix, est remise en cause par l'émergence des territoires communaux. Cependant, les relations réticulaires qui les caractérisent sont toujours d'actualité et la demande des élus communaux est très forte pour que ces liens privilégiés entre les villages et les communautés de migrants se renouvellent, et ce sur le mode de l'adhérence au territoire communal. En cela, la place des réseaux sociaux dans le processus de territorialisation semble essentielle pour ces espaces mobiles, et leur partage, dans la continuité des espaces sociaux.

\section{Notes}

1. Structure chargée de la mise en ceuvre de la réforme de décentralisation, entre 1993 et 2001.

2. Mahamet Timera, Les Soninkés en France. D'une histoire à l'autre, Paris, Karthala, 1996, 233 p.

3. Patrick Gonin, "Migrations développement : les utopies des années quatre-vingt-dix", in Institut Panos (éd.), D'un voyage à l'autre. Des voix de l'immigration pour un développement pluriel, Paris, Karthala, 2001, pp. 25-46.

4. Adrian Adams, Le long voyage des gens du fleuve, Paris, François Maspéro, 1977, 222 p. 